Type of the Paper: Research Article

\title{
Title: Components of Diet Quality Explain the Association between Acculturation and Increased Body Mass Index in Mexican Americans
}

\author{
Yilin Yoshida ${ }^{1}$, Richard Scribner ${ }^{2}$, Liwei Chen ${ }^{3}$, Stephanie Broyles ${ }^{4,5}$, Stephen Phillippi ${ }^{4}$, \\ Eduardo J. Simoes ${ }^{1}$, and Tung-Sung Tseng ${ }^{4}$ * \\ ${ }^{1}$ Department of Health Management and Informatics, School of Medicine, University of Missouri \\ 2 Program of Epidemiology, School of Public Health, Louisiana State University Health Sciences \\ Center at New Orleans \\ ${ }^{3}$ Department of Public Health Sciences, Clemson University \\ 4 Program of Behavioral and Community Health Sciences, School of Public Health, Louisiana State \\ University Health Sciences Center at New Orleans \\ ${ }_{5}^{5}$ Pennington Biomedical Research Center* \\ Correspondence Email: ttseng@lsuhsc.edu. Mailing address: 2020 Gravier Street, 3rd Floor New Orleans, LA \\ 70112. Phone: 504-568-6022.
}

\begin{abstract}
:
Background and Objectives: Acculturation is associated with excessive weight gain among immigrants to the U.S. Whether dietary factors mediate this association is unclear. This study aimed to examine whether overall diet quality or specific component(s) of diet quality mediate the association between acculturation and Body Mass Index (BMI) among Mexican American (MA) men and women.
\end{abstract}

Material and Methods: This is a secondary data analysis using dietary intake data from 24-hour dietary recalls, measured height and weight, and self-reported acculturation from the National Health and Nutrition Examination Survey (NHANES) cycles 1999-2000 to 2011-2012. A total of 6848 MA adults ( $\geq 20 \mathrm{yrs})$ with reliable dietary recall status and body measures were included in the study. Path analyses was performed in Mplus with complex survey design effects adjusted.

Results: HEI components of whole grains and sodium were found to play meditating roles in the acculturation-BMI association, and their effects on BMI were opposing [indirect effect were -0.01 (SE 0.00) and $0.02(0.01)$, respectively]. In gender-specific analysis, sodium [0.01 (0.00)] was a significant mediator only in MA men; whereas, whole grains [-0.01 (0.00)] was a significant mediator only in MA women.

Conclusions: HEI components of whole grains and sodium appeared to be significant dietary mediators in the acculturation-BMI association. Understanding the variations of dietary components and their relationship with acculturation as well as BMI is useful for developing dietary interventions and obesity reduction.

Keywords: 1 Acculturation; 2 Diet Quality; 3 Healthy Eating Index; 4 Body Mass Index; 5 Mexican Americans; 6 NHANES 


\section{Introduction}

The burden of obesity in Mexican Americans (MAs) varies by the level of acculturation [1-6] a process in which immigrants adopt the customs and behaviors of a new culture [7]. Research showed that MAs' risks for obesity increase with the increase of acculturation [1-6]. Compared to U.S.-born individuals, foreign-born MAs have lower body weight, regardless of their socioeconomic status [1, 3-5]. Additionally, duration of U.S. residency and English fluency were also found to be positively associated with obesity $[2,3,6]$.

The differences in obesity status by acculturation are likely to be attributable to changes in lifestyles, including dietary changes [8,9]. Current findings regarding the relationship between acculturation and diet are equivocal. Some studies suggested that acculturation to U.S. society was associated with adverse nutritional profiles and unhealthful dietary behavior $[9,10]$. Such that, a higher degree of acculturation was related to lower intake of fruit and vegetables, but higher intake of total fat, saturated fat, sodium, sugar, and sugar-sweetened beverages [9, 11]. Acculturation was also associated with more consumption of commercially prepared foods $[9,11,12]$, which often are high in sodium, sugar, and saturated fat, but low in fibers [13]. Others, however, demonstrated protective effects of acculturation on aspects of diet, such as increased intake of whole- or multigrains products $[12,14]$. Compared to individuals living in Mexico, acculturated MAs are more likely to take advantage of the wide varieties of whole-grain foods in the U.S. food environment [14]. The relationship between acculturation and diet seems complex as the previous studies suggest opposite association and often only involved outcome of a single nutrient or a food group. Further investigation equipped with comprehensive dietary assessment that enables evaluating diet quality and variety of dietary components is therefore warranted.

Diet quality reflects the combination of nutrients and foods, rather than inclusion of food(s) or nutrient(s) in isolation from one another or caloric intake exclusively [17]. Studies have shown a possible link between diet quality and weight outcomes [15-17]. The Healthy Eating Index (HEI) is developed by the U.S. Department of Agriculture for monitoring dietary intake and nutrition promotion activities for the U.S. population [18]. The total HEI score examines the overall dietary quality; while the component scores used to calculate the total HEI score can be used to study components of dietary intake and their relationship with obesity or disease risk [19]. Previous research has shown inverse associations between the HEI and risks for obesity, central obesity and obesity-related conditions in general adult populations [16, 19-22]. A low total HEI score was associated with overweight and obesity among U.S. adults including Mas [19, 21]. A high consumption of proteins, sodium and empty calories was associated with increased risk for overweight and obesity [16].

While evidence has undertaken the association between the HEI and weight outcomes [1622], as well as the association between acculturation and diet quality or weight outcomes $[3,6,7]$, little is known whether the overall diet quality or specific component(s) of diet quality mediates the association between acculturation and body mass index (BMI). Using weight and dietary data in a national sample of MAs, the current study aimed to 1) examine whether the overall diet quality (i.e., HEI total score) mediates the association between acculturation and BMI; 2) which aspect(s) of diet quality (i.e., HEI component scores) mediate the association between acculturation and BMI among MAs. Gender disparities of obesity among MAs exist [23-26]. While national age-adjusted prevalence of overall obesity were $45 \%$ vs $47 \%$ for MA women and men, respectively; in California and on Texas border, researchers reported MA men have almost the same likelihood to be obese as their women counterparts [23-25]. In addition, effect of acculturation on dietary changes and weight gain varies between genders [24, 26]. For example, MA men were reported to consume more western style fast food than women due to acculturation [24, 26]. For these reasons, we performed gender-specific analyses. 


\section{Materials and Methods}

Data. Cross-sectional data from the Continuous NHANES cycle 1999-2000 to cycle 2010-2012 were obtained for the analysis. The NHANES is a complex, multistage probability sample of U.S. noninstitutionalized civilians that started in 1999 (detailed information NHANES is described somewhere else and the data can be found on NHANES website) [26]. The National Center for Health Statistics Research Ethnic Review Board approved NHANES, and informed consent was obtained from all participants [27]. Data from the United States Department of Agriculture (USDA) Food Pattern Equivalents Databases (FPED) were utilized to translate NHANES dietary data into equivalent servings of the major food groups and subgroups according to the HEI2010.

Measures. Weight status. The primary outcome, BMI, was calculated as weight divided by the square of height $(\mathrm{kg} / \mathrm{m} 2)$. The body measurement data was collected in the Mobile Examination Center (MEC), by trained health technicians. NHANES used digital scales and stadiometers to measure height and weight. Detailed measurement procedures were documented somewhere else [28].

Acculturation. The main predictor of interest, acculturation was constructed as an acculturation score, which was based on three proxy measures: country of birth, language spoken at home, and length of time in the U.S. Combining country of birth and length of time in the U.S., a score of 0-3 score was assigned based on four categories ( $3=$ U.S. born, $2=$ foreign born and lived in the U.S. $\geq 20$ years, $1=$ foreign born and lived in the U.S. 10-19 years, $0=$ foreign born and lived in the U.S. $<10$ years). A score of $0-2$ was assigned to language spoken at home (2=English only or pro-English, $1=$ both equally, $0=S$ panish or pro-Spanish). These scores were summed to yield a total acculturation score, ranging from 0 (least acculturated) to 5 (most acculturated). This is a validated scale that has been tested in Hispanic and Chinese populations in the U.S. [28]. Instead of using the three components as separate variables, it is argued that an acculturation score gives a more accurate representation of acculturation status than each independent indicator, in that these characteristics are usually clustered within an individual and they are inseparable [29].

Diet quality. The study mediators, the HEI2010 scores, were composed based on dietary intake data from NHANES in-person 24-hour recall interview. Detailed descriptions of the dietary recalls and data collection are available elsewhere [30]. FPED from USDA then translated dietary recall data into equivalent servings of the major food groups and subgroups. The HEI2010 has 12 categories, including 9 "adequacy" (total vegetables, greens and beans, total fruit, whole fruit, total proteins, seafood and plant proteins, whole grains, dairy, and fatty acids) and 3 "moderation" (refined grains, sodium and empty calories) components. For all components, higher scores indicate closer conformance with dietary guidelines [18]. The scores assigned for the 9 adequacy and the 3 (reverse-scored) moderation components were summed to yield a total score ranging from 1 to 100 , with higher score indicating a better diet quality [18].

Covariates. The following sociodemographic covariates were considered: gender, age (20-40, 41-60, or $>60$ ), education (less than high school, high school or equivalent, or more than high school), marital status (yes or no), poverty-income ratio (PIR $<1,1 \leq \mathrm{PIR}<3$, or $\mathrm{PIR} \geq 3$ ), and insurance coverages (public insurance including Medicare, Medicaid or other forms of government insurance, private insurance, or no health insurance). Potential confounding effects from other behavioral factors were also adjusted, such as smoking (never, former, or current), alcohol drinking (never, former, or current), and physical activity (PA). PA was assessed by using the physical activity questionnaire (PAQ) items employed in NHANES. Participants were asked their engagement in moderate-tovigorous physical activity (MVPA) during the past 30 days related to transportation, household/domestic tasks, and leisure-time activities. Their responses were translated to minutes/week of MVPA. Each participant's combined weekly duration of MVPA were grouped 
into one of two categories ( $<150$ or $\geq 150$ minutes/week) based upon their achievement of the current activity guidelines [31]. Lastly, Data Release Number (SDDSRVRY) was taken into account in the analysis to adjust for potential different distributions of sampled populations in different survey cycles.

Analysis. A final 6847 Mexican Americans $\geq 20$ years with non-missing dietary and non-pregnancy status were included in the study. Path analyses were used to test whether the HEI2010 mediates the association between acculturation and BMI. Because whole fruit, greens and beans, seafood and plant proteins are parts of total fruit, total vegetables, and total proteins, respectively, they were excluded from the analysis to avoid collinearity. Acculturation and a set of sociodemographic factors were modeled as being correlated and as having both direct and indirect (through mediators, HEI component scores) effects on the dependent variable, BMI. Mediation proportion [indirect effect/ (indirect+direct effects)] $\times 100$ were used to determine the mediation effect size.32 A mediation proportion of $10 \%$ or more is considered as statistically significant mediation effect, and $5 \%$ to $10 \%$ as moderate mediation effect. 33 All analyses were conducted using Mplus version 7.3 (Muthen \& Muthen, Los Angeles, CA) with complex survey design effects adjusted.

\section{Results}

Table 1 presents descriptive results of the study. The mean acculturation index was 2.4, mean BMI was 29, and mean HEI total score was 29.1 for MAs in NHANES cycles 1999-2000 to 2010-2012. In gender-specific analyses, women appeared less likely to be current smokers (12\% vs. 27\%, $\mathrm{P}<.001)$ and current drinkers (53\% vs. 79\%, $\mathrm{P}<.001)$, but more likely to achieve the Physical Activity Guidelines for Americans (PAGA) (63\% vs. 52\%, P<.001), and have better diet quality (HEI total score 51.1 vs. 48.4, $\mathrm{P}<.001)$. However, in comparison to men, women were more likely to be acculturated (mean acculturation index 2.6 vs. 2.3, P<.001) and to have higher BMI (mean BMI 29.5 vs. 28.6, $\mathrm{P}<.001)$.

Table 1. Sociodemographic and Behavioral Characteristics by Gender Group among MAs, NHANES 1999-2012

\begin{tabular}{|c|c|c|c|c|}
\hline & All & Men & Women & \\
\hline n (\%) & & & & $\mathrm{P}$ \\
\hline Age (years) & & & & ** \\
\hline $20-40$ & $3096(61.5)$ & $1382(63.6)$ & $1590(59.2)$ & \\
\hline $41-60$ & $1948(29.0)$ & $941(28.5)$ & $940(29.6)$ & \\
\hline$>60$ & $1803(9.5)$ & $788(8.0)$ & $899(11.2)$ & \\
\hline Education & & & & ** \\
\hline Less than high school & $4047(53.0)$ & $1896(55.1)$ & $1954(50.6)$ & \\
\hline High school or equiv. & 1235 (20.7) & $563(21.4)$ & $622(20.0)$ & \\
\hline More than high school & $1550(26.3)$ & $649(23.4)$ & $844(29.4)$ & \\
\hline Income $^{1}$ & & & & ** \\
\hline $\mathrm{PIR}<1.0$ & 1929 (31.3) & $830(29.1)$ & $1026(33.7)$ & \\
\hline $1 \leq \mathrm{PIR}<3$ & $2039(47.8)$ & $1413(49.9)$ & $1422(45.5)$ & \\
\hline $\mathrm{PIR} \geq 3$ & $1550(20.9)$ & $567(21.9)$ & $587(20.8)$ & \\
\hline Marital status & & & & $*$ \\
\hline Married & $3971(56.6)$ & $1953(58.5)$ & $1849(54.5)$ & \\
\hline Health insurance & & & & \\
\hline
\end{tabular}




\begin{tabular}{|c|c|c|c|c|}
\hline No & $2944(51.1)$ & $1451(54.9)$ & $1387(46.9)$ & $* *$ \\
\hline Public & $1544(14.0)$ & $573(9.9)$ & $888(18.6)$ & \\
\hline Private & $2257(34.9)$ & $1052(35.2)$ & $1106(34.5)$ & \\
\hline Smoking & & & & $* *$ \\
\hline Non-smoker & $4120(61.5)$ & $1391(49.4)$ & $2539(74.9)$ & \\
\hline Former & $1494(18.6)$ & $923(23.5)$ & $506(13.2)$ & \\
\hline Current & $1224(19.9)$ & $795(27.1)$ & $378(12.0)$ & \\
\hline Alcohol drinking & & & & ** \\
\hline Non-drinker & $997(15.4)$ & $111(4.4)$ & $886(27.6)$ & \\
\hline Former & $1164(17.7)$ & $555(16.5)$ & $609(19.2)$ & \\
\hline Current & $3437(66.9)$ & $2001(79.2)$ & $1436(53.2)$ & \\
\hline Physical activity $^{2}$ & & & & $* *$ \\
\hline Met PAGA ( $\geq 150 \mathrm{~min} / \mathrm{wk})$ & $4084(56.7)$ & $1703(51.5)$ & $2192(62.5)$ & \\
\hline \multicolumn{5}{|l|}{ Mean score (SE) } \\
\hline Acculturation $(\text { index } 0-5)^{3}$ & $2.4(0.1)$ & $2.3(0.1)$ & $2.6(0.1)$ & $* *$ \\
\hline $\mathrm{BMI}^{4}$ & $29.0(0.1)$ & $28.6(0.2)$ & $29.5(0.2)$ & ** \\
\hline HEI total score $(0-100)^{5}$ & $49.7(0.3)$ & $48.4(0.3)$ & $51.1(0.4)$ & ** \\
\hline Total vegetables $(0-5)^{5}$ & $3.5(0.0)$ & $3.4(0.0)$ & $3.6(0.0)$ & ** \\
\hline Greens and beans $(0-5)^{5}$ & $2.1(0.0)$ & $2.1(0.0)$ & $2.0(0.1)$ & ** \\
\hline Total fruit $(0-5)^{5}$ & $2.1(0.0)$ & $1.9(0.1)$ & $2.4(0.0)$ & ** \\
\hline Whole fruit $(0-5)^{5}$ & $2.0(0.0)$ & $1.8(0.1)$ & $2.2(0.1)$ & ** \\
\hline Whole grains $(0-10)^{5}$ & $1.4(0.0)$ & $1.1(0.1)$ & $1.6(0.1)$ & ** \\
\hline Total dairy $(0-10)^{5}$ & $4.7(0.1)$ & $4.2(0.1)$ & $5.2(0.1)$ & ** \\
\hline Total proteins $(0-5)^{5}$ & $4.4(0.0)$ & $4.5(0.0)$ & $4.3(0.0)$ & ** \\
\hline Seafood \& plant protein $(0-5)^{5}$ & $2.0(0.0)$ & $1.9(0.1)$ & $2.0(0.0)$ & * \\
\hline Fatty acids $(0-10)^{5}$ & $5.2(0.1)$ & $5.2(0.1)$ & $5.1(0.1)$ & ** \\
\hline Sodium $(0-10)^{5}$ & $5.4(0.1)$ & $4.5(0.1)$ & $5.2(0.1)$ & ** \\
\hline Refined grains $(0-10)^{5}$ & $4.6(0.1)$ & $4.6(0.1)$ & $4.7(0.1)$ & 0.06 \\
\hline Empty calories (0-20) 5,6 & $12.4(0.1)$ & $12.1(0.1)$ & $12.8(0.2)$ & ** \\
\hline
\end{tabular}

${ }^{1}$ PIR: Poverty-to-Income Ratio

2 PAGA: Physical Activity Guidelines for Americans. According to PAGA, moderate-intensity physical activities of $\geq 150$ minutes per week is needed.

${ }^{3}$ Acculturation is an index of 0-5 ( 0 is the lowest acculturation and 5 is the highest acculturation). The index derived from information of length of stay, nativity, and language spoken at home.

${ }^{4}$ BMI: Body Mass Index (kg/m2)

${ }^{5}$ Maximum score for each HEI category is in paraphrase; higher score means better quality.

${ }^{6}$ Empty calories are from solid fats, alcohol, and added sugars.

missing: age 307; education 319; PIR 1005; married 430; insurance status 390; PA 1249; smoking 315

${ }^{*}=\mathrm{P}<0.05,{ }^{* *}=\mathrm{P}<0.001 . \mathrm{P}$ is from chi-square test for difference between genders.

Table 2 and Figure 1.A and 1.B present results from the first set of path analyses - the relationship between acculturation and BMI through the overall diet quality (i.e., HEI total score). Among all MAs, the total effect of acculturation on BMI before adjusting the mediator was significant [total effect a=.56 (SE .06), $\mathrm{P}<.001$ ] (Table 2). The direct effect of acculturation on BMI (i.e., independent of all measured mediators and confounders) remained significant [direct effect a1 $=.55$ (SE.06), $\mathrm{P}<.001$ ] (Table 2). However, the indirect effect of HEI total score was not significant [indirect effect a2*a3=.02 (SE .01), $\mathrm{P}=0.06$ ] (Table 2). The size of the indirect effect was only $3 \%$ of the total 
181

182

183

184

185

186

187

188

189

190

191

192

193

194

195

196

197

198

199

200

201

202

203

effect. This effect size was small considering the criterion of significance is $10 \% 32$. In genderspecific analyses, total and direct effects of acculturation on BMI were significant in both gender groups (all P-values <.001) (Table 2 and Figure 1.A. and 1.B.). However, the indirect effects of HEI total score was not significant in either group $(\mathrm{P}=0.21$ and $\mathrm{P}=.17$ for men and women, respectively) (Table 2 and Figure 1.A. and 1.B.).

Table 2. Path Analysis for the Association between Acculturation and BMI through HEI2010 Total Score (Coefficient, SE)

\begin{tabular}{llll}
\hline & All & Men & Women \\
\hline${\text { Total effect (a) }{ }^{1}}^{\text {Direct effect (a1) }{ }^{2}}$ & $0.56(0.06)^{* *}$ & $0.62(0.08)^{* *}$ & $0.48(0.08)^{* *}$ \\
\hline${\text { Indirect effect }\left(\mathbf{a} 2^{*} \text { a3) }{ }^{3}\right.}^{{ }^{*}}$ & $0.55(0.06)^{* *}$ & $0.61(0.09)^{* *}$ & $0.46(0.01)^{* *}$ \\
\hline
\end{tabular}

${ }^{1}$ Total effect is the effect of acculturation on BMI before adjusting for the mediator (i.e., HEI total score) and all confounders, including age, gender, education, income, marriage status, insurance status, smoking status, alcohol drinking status, and physical activity.

2 Direct effect is the effect of acculturation on BMI after adjusting for the mediator and all confounders.

${ }^{3}$ Indirect effect is the mediating effect of HEI total score on the association between acculturation and BMI after adjusting for all confounders.

${ }^{*}=\mathrm{P}<0.05,{ }^{* *}=\mathrm{P}<0.001$. $\mathrm{P}$ is from the bootstrap approach.

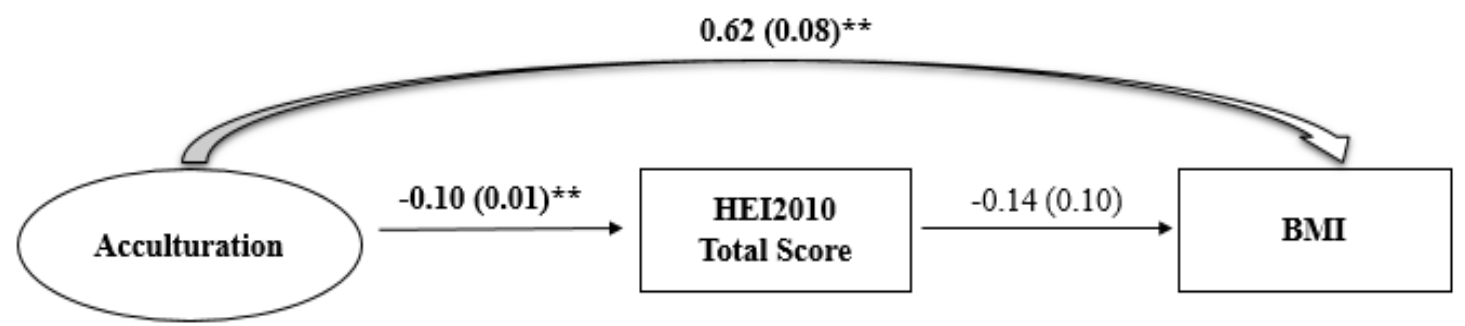

${ }^{*}=\mathrm{P}<.05,{ }^{* *}=\mathrm{P}<.001 . \mathrm{P}$ is from the bootstrap approach.

Figure 1.A. Mediating Effect of HEI2010 Total Score in the Association between Acculturation and BMI among MA Men

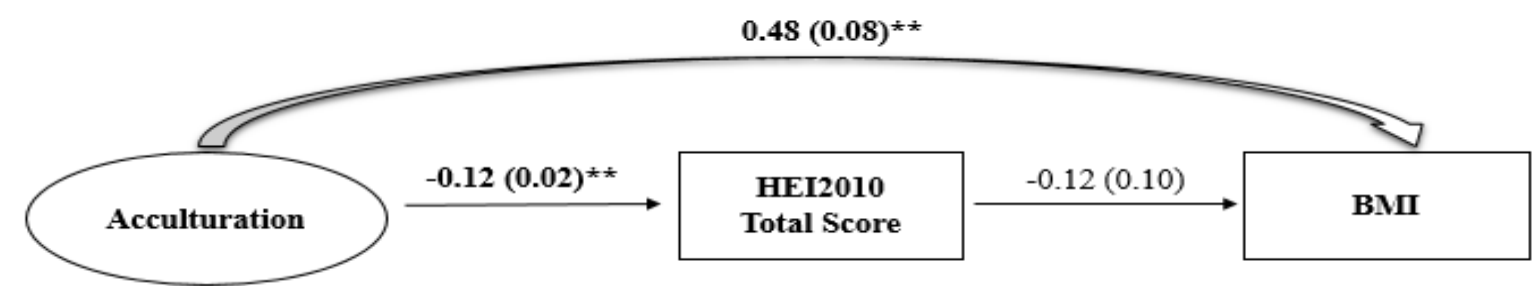

$*=\mathrm{P}<.05, * *=\mathrm{P}<.001 . \mathrm{P}$ is from the bootstrap approach.

Figure 1.B. Mediating Effect of HEI2010 Total Score on the Association between Acculturation and BMI among MA Women 
Table 3 and Figure 2.A. and 2.B. present results from the second set of mediation analyses, where HEI component scores mediate the relationship between acculturation and BMI were examined. Among all MAs, the total effect of acculturation on BMI [total effect a=.59 (SE .06), $\mathrm{P}<.001$ ] was significant (Table 3). After adjusting for all covariates, scores of whole grains [indirect effect a2*a3=-.01 (SE .00), P<.05] and sodium [indirect effect a2*a3=.02 (SE .01), P<.05] had statistically significant indirect effects on BMI (Table 3). The direct effect of acculturation on BMI, independent of all measured mediators and confounders remained significant [direct effect a1=.58 (SE.06), P<.001] (Table 3).

Table 3. Path Analysis for the Association between Acculturation and BMI through HEI-2010 Component Scores (Coefficient, SE)

\begin{tabular}{llll}
\hline HEI components & All & Men & Women \\
\hline Total effect (a) ${ }^{1}$ & $0.59(0.06)^{* *}$ & $0.66(0.10)^{* *}$ & $0.50(0.07)^{* *}$ \\
\hline${\text { Direct effect (a1) }{ }^{2}}^{*}$ & $0.58(0.06)^{* *}$ & $0.65(0.09)^{* *}$ & $0.48(0.07)^{* *}$ \\
\hline Indirect effect from each & & & \\
component (a2*a3) ${ }^{3}$ & & & \\
\hline Total vegetables & $-0.00(0.01)$ & $-0.01(0.01)$ & $0.00(0.01)$ \\
\hline Total fruit & $0.01(0.01)$ & $0.01(0.01)$ & $0.01(0.02)$ \\
\hline Whole grains & $-0.01(0.00)^{*}$ & $-0.10(0.01)$ & $-0.01(0.00)^{*}$ \\
\hline Total dairy & $0.00(0.00)$ & $0.00(0.00)$ & $-0.01(0.01)$ \\
\hline Total proteins & $-0.01(0.00)$ & $-0.01(0.01)$ & $-0.01(0.01)$ \\
\hline Fatty acids & $0.00(0.00)$ & $0.01(0.01)$ & $0.00(0.00)$ \\
\hline Sodium & $0.02(0.01)^{*}$ & $0.01(0.00)^{*}$ & $0.02(0.01)$ \\
\hline Refined grains & $-0.00(0.01)$ & $-0.00(0.01)$ & $-0.01(0.01)$ \\
\hline Empty calories & $-0.01(0.01)$ & $-0.01(0.01)$ & $-0.00(0.01)$ \\
\hline
\end{tabular}

${ }^{1}$ Total effect is the effect of acculturation on BMI before adjusting for the mediators (i.e., HEI component scores) and all confounders, including age, gender, education, income, marriage status, insurance status, smoking status, alcohol drinking status, and physical activity.

${ }^{2}$ Direct effect is the effect of acculturation on BMI after adjusting for the mediators and all confounders.

${ }^{3}$ Indirect effect is the mediating effect of each HEI component score on the association between acculturation and BMI after adjusting for all confounders.

${ }^{*}=\mathrm{P}<0.05,{ }^{* *}=\mathrm{P}<0.001$. $\mathrm{P}$ is from the bootstrap approach. 


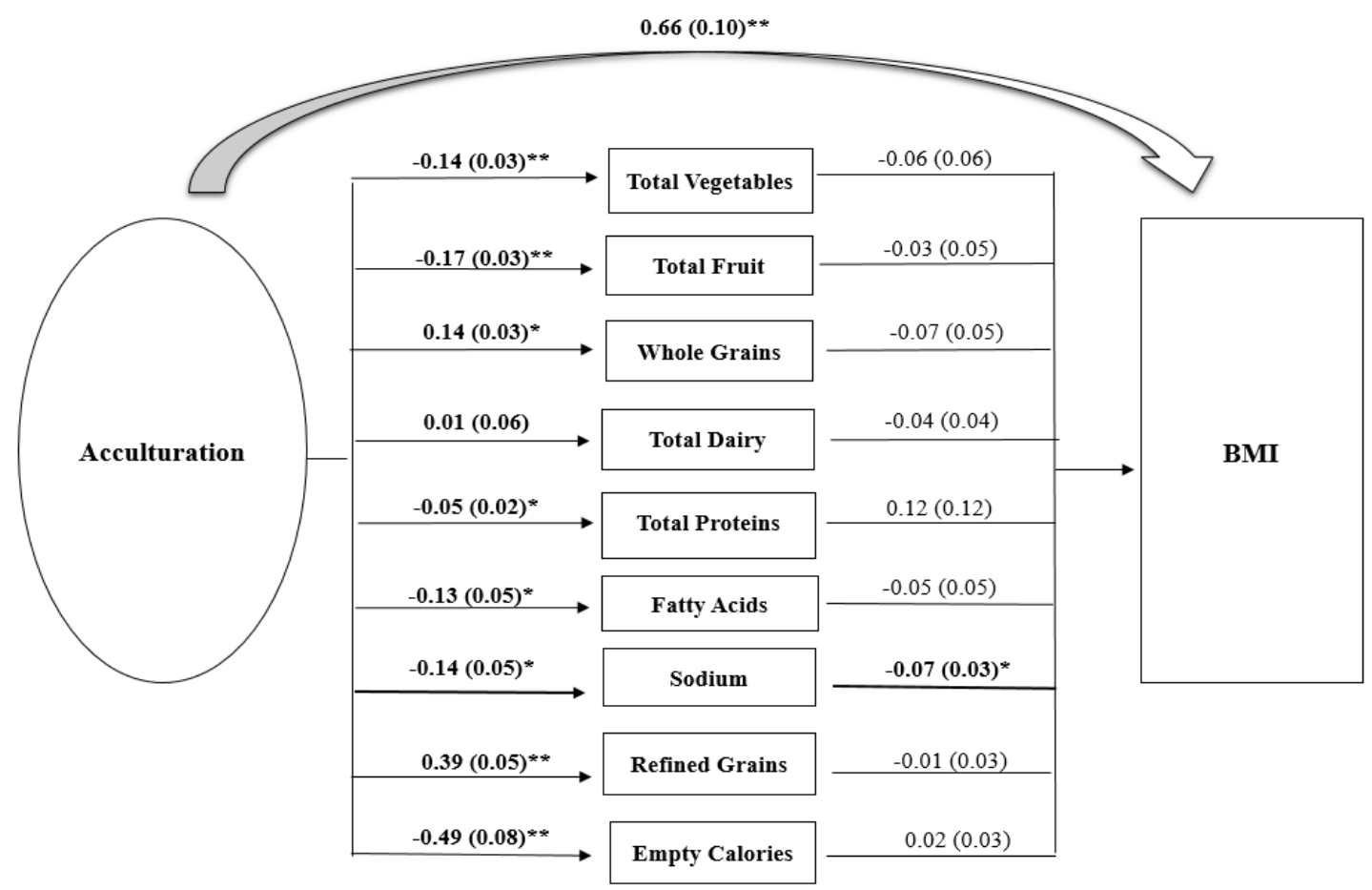

$232 *=\mathrm{P}<.05,{ }^{* *}=\mathrm{P}<.001 . \mathrm{P}$ is from the bootstrap approach.

233 Figure 2.A. Mediating Effect of HEI2010 Component Scores on the Association between 234 Acculturation and BMI among MA Men

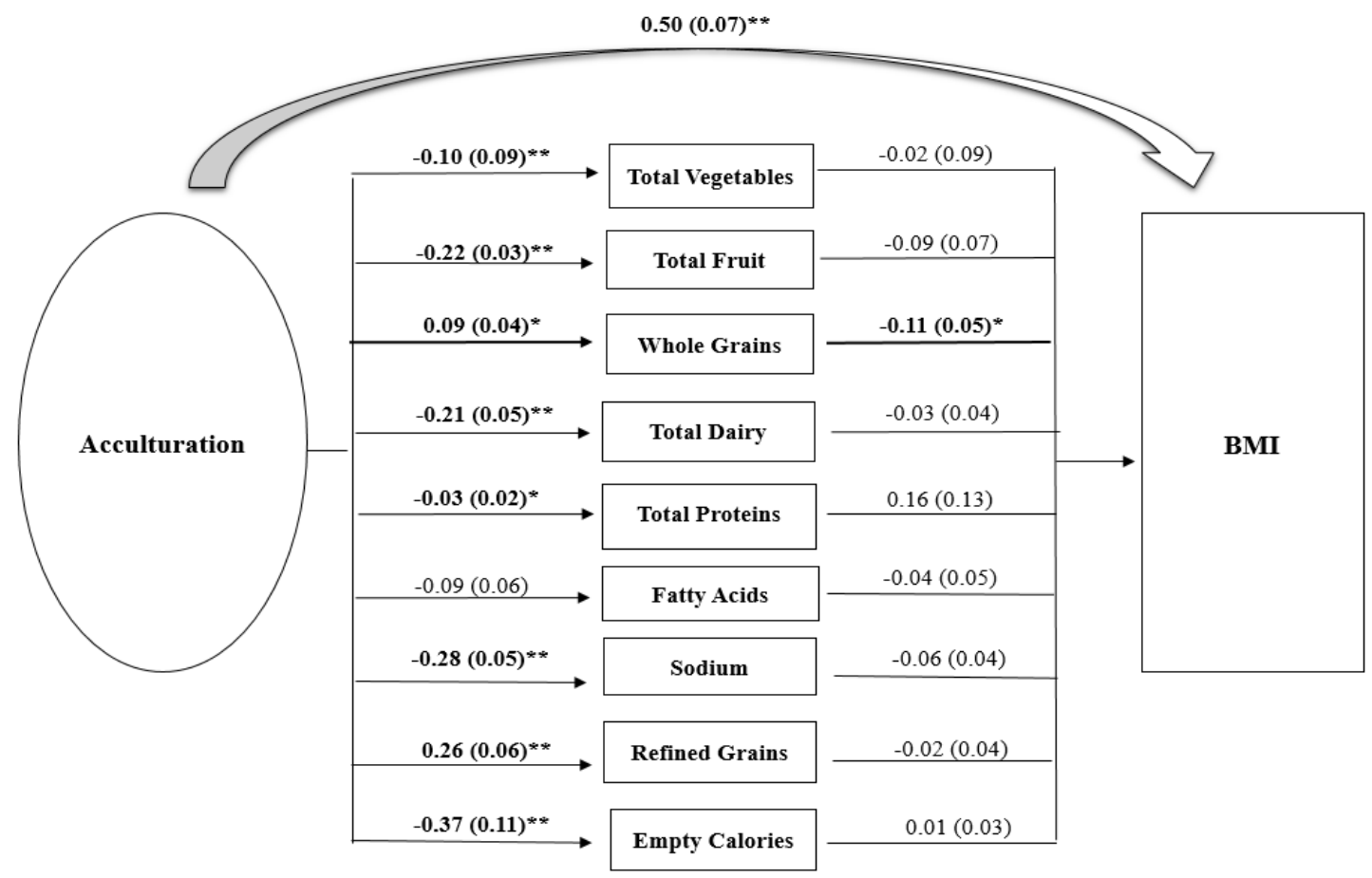

$237 *=\mathrm{P}<.05, * *=\mathrm{P}<.001 . \mathrm{P}$ is from the bootstrap approach.

238 Figure 2.B. Mediating Effect of HEI2010 Component Scores on the Association between

239 Acculturation and BMI among MA Women 
When stratifying gender groups, in men, sodium appeared have a statistically significant mediating effect in the association between acculturation and BMI [indirect effect a2*a3=.01 (SE .00$)$, $\mathrm{P}<$.05] (Table 3 and Figure 2.A.). The score of whole grains, however, was the only component that significantly mediated the association between acculturation and BMI [indirect effect a2*a3=- .01 (SE $.00), \mathrm{P}<.05$ ] in MA women (Table 3 and Figure 2.B.). The overall and gender-specific models fit well given the model fit indices were within threshold levels (i.e., Root mean square error approximation (RMSEA) <0.1; Standardized root mean square residual (SRMSR) <.08; Comparative fit index/Tucker Lewis Index (CFI/TLI)>.9) (Tables are not presented).

\section{Discussion}

The present study examined whether the HEI total or component scores mediate the association between acculturation and BMI. To our knowledge, this study is among the first that explored these relationships through path analyses. The study found that the HEI total score was not a significant mediator of the acculturation-BMI association. However, HEI components of whole grains and sodium were found to play a significant mediating role in the relationship of the interest. The findings suggest that acculturation is positively associated with a higher score of whole grains (reflects a higher level intake of whole grains) among men; it was also associated with a lower score of sodium (reflects a higher level intake of sodium) among women. In other words, compared to those who scored lower in acculturation, MA men with a higher degree of acculturation may have a diet higher in whole grains, which is protective to BMI. Whereas among women, compared to those less acculturated, those who are more acculturated may have a diet higher in sodium, which is associated with higher BMI.

The association between acculturation and increased consumption of whole grains was supported by studies targeting Hispanic populations in the U.S., including Mas [34, 35]. According to Winkley et al., a greater level of acculturation, as measured by English fluency, was associated with increased intake of whole-grain foods [32]. Liu et al. also found that generation status, reflecting the level of acculturation, was associated with increased intake of whole grains, where the second and third generations of MAs have significantly higher intake of whole grains as compared to the first generation [35]. A significant inverse relationship between the intake of whole grains and BMI has been reported previously [36]. Whole grains provide dietary fibers, which have appetite-suppressant qualities that helps one feels satiated, thereby preventing unhealthy snacking [37]. Moreover, the health effect of whole grains is also attributable to micronutrients, antioxidants and non-nutritive dietary constituents such as phyto-estrogens in wheat bran, and beta-glucans in oats [38].

With respect to sodium intake, increased acculturation was found to be associated with its increased consumption [11,41,44]. Behaviors associated with sodium consumption include eatingaway-from-home, consumption of meals at fast-food outlets and consumption of salty snacks [11]. Researchers have studied the link between sodium intake and weight gain [11, 39-41] and there are several hypotheses for this association. Karppanen and Mervaala suggested that increasing intake of sodium obligatorily produced a progressive increase in thirst [40]. The increase in salt intake may contribute to increased intake of sugar sweetened or alcoholic beverages, which is a source of excessive calories. Alternatively, a high level of sodium intake is correlated with a high consumption of processed foods, which are generally of higher energy density [11, 39]. Most sodium in the U.S. diet comes from commercially processed and restaurant foods [40]. According to U.S. Centers for Disease Control and Prevention, more than seventy-five percent of sodium Americans consume comes from processed and restaurant foods - not the salt shaker [43]. It has been observed that many MAs favor ready-made processed foods and eating out because of the 
fast-paced life in the U.S., or the pressure to integrate into American society by eating American style fast-food [44]. Consequently, sodium intake may be a marker for consumption of unhealthy processed foods, which may explain the link between high level of sodium intake and elevated BMI.

No study that we are aware of that has conducted a similar mediation analysis by gender groups. Using a gender-mixed sample, Flores et al. found that "refined foods and sweets" and "diverse" dietary patterns were associated with increased risks for overweight and obesity, in comparison to "traditional" dietary pattern [45]. It was reported that immigrant men consume fast food more frequently than their female counterparts, possibly due to their preference of its ease and convenience, and their cooking and time limitations [46]. Fast foods that contain excessive amount of sodium and other unhealthy ingredients that are associated with weight gain. Wolongevicz et al. found that a diet high in refined carbohydrates was associated with obesity in a women-only sample [47]. Other studies suggested that intake of whole grains was related to lower odds of obesity in both men and women [47, 48], though sample characteristics, dietary measurements and analytical methods in those studies are not comparable to the current one. The limited findings regarding specific HEI components and weight outcomes by gender group warrant more genderspecific analysis to explore the differences regarding diet quality and its association with weight outcomes.

HEI total score was not a significant mediator in the acculturation-BMI association in the current study. It was previously reported that HEI total score was inversely associated with body fat in a sample of young men aged 21 to 35 years old [14]. The absence of significant difference may be due to the different sample composition where the current one includes adults from all age groups and focuses exclusively on MAs. Further, a challenging issue of using the HEI2010 total score to predict BMI in MAs is that the relative contribution of the components to the total score is possibly different in MAs from the general U.S. population [49]. It would be better to ascribe greater weights to those items that affect BMI of MAs to a greater extent [49]. Research is therefore needed to study the health effect of different HEI components in MAs and assign proper weights to them.

The statistically insignificant mediating effect of the HEI total score may be also due to other unmeasured factors. Such factors could include the following: beliefs and norms regarding ideal body images that are related to U.S. and/or Mexican culture [50]; food insecurity, a state in which availability of nutritionally adequate foods or one's ability to acquire food, is limited or uncertain [51, 52]; or other contextual factors, such as density of a Hispanic/Latino population in a given neighborhood, the history of migration to the U.S. and social network influences [11]. Indeed, it is difficult to draw a clear pathway of acculturation-diet quality-BMI given that acculturation is influenced, in part, by these contextual factors [11]. The close relationship between diet and BMI, as well as the significant impact of acculturation on diet and weight changes, compelled us to examine the acculturation-BMI path through diet quality. However, the study findings may also indicate unexplained mechanism between acculturation and BMI. Additional studies are warranted to assess the relative contributions of psychosocial and contextual factors of acculturation that account for changes in BMI.

The study has several limitations. The study used cross-sectional data, which precludes assessment of changes in diet quality and BMI in MAs over time. It is possible that some foreignborn participants had nutritionally poor diets and were overweight or obese before moving to the U.S. The nutritional transition has been observed in Mexico, where processed food consumption and away-from-home food intakes are rapidly increasing in urban areas and obesity rates and related chronic conditions are following the trend [53]. A causal relationship between acculturation and dietary changes cannot be established due to the cross-sectional nature of the data. In addition, arbitrary choices made in scoring HEI components may hamper its ability to predict risks for 
chronic diseases [49]. For example, the HEI-2010 considers alcohol above a threshold level indicative of moderate drinking as empty calories. This approach does not directly address other potential positive and negative biological effects of alcohol, nor does it account for specific questions related to frequency of consumption and amounts consumed on drinking occasions. This probably can serve as a reason why we did not detect the significant mediating effect of empty calorie as expected. Further, the measure of acculturation was based on proxies of language, length of stay and nativity. Even though they are well-validated measures in the literature and only available acculturation information in NHANES data, they may not capture all aspects of culture adaptation that impact diet and weight. Future studies should incorporate more comprehensive scales to study the relationships between acculturation, diet quality and BMI. Further, potential biases may exist in measuring diet. Underreporting by participants is common in nutritional studies, especially among those who are overweight or obese [54]; thus, the potential bias toward the null, resulting from underreporting was acknowledged.

\section{Conclusions}

The current study found HEI components of whole grains and sodium, appear to be significant dietary mediators in the acculturation-BMI association. These results present insights with respect to heterogeneity in the relative influence of HEI components on the path between acculturation and BMI, adjusting sociodemographic and behavioral factors simultaneously. More studies, especially perspective studies, are needed to further clarify the path of acculturation, diet quality and BMI.

Acculturation is associated with increased consumption of whole grains, which may contribute to a better weight status. Health messages may be important in delivering information to those who are less acculturated with traditional preference for refined grains. The benefits of including whole- and multi-grain foods in one's diet should be empathized. Another strategy may be to increase awareness of recent immigrants to the wide varieties of whole-grain alternatives, such as whole-grain tortilla and whole-grain bread by highlighting their health benefits and flavor.

Promoting a moderation on sodium intake is also essential as it was found to be another dietary mediator to acculturation and BMI. Promoting home cooking as a strategy for reducing sodium intake may be particularly beneficial for MA men, who tend to consume more fast food and have poorer cooking skills compared to MA women [44, 55]. It is also important to enhance the understanding of food labels, of nutritional merits of different foods and of food preparation methods and to incorporate this information into the dietary interventions. A regulatory approach supporting the food industry in the reformulation of foods would also be necessary [56]. If these efforts were implemented, they would reduce barriers for meeting the sodium intake guideline when individuals consume commercially prepared foods.

Author Contributions: Conceptualization, Y.Y.; Methodology, Y.Y., T.S.T.; Software, Y.Y.; Validation, Y.Y., T.S.T., R.S., L.W., S.B., S.P., and E.J.S; Analysis, Y.Y.; Writing-Original Draft Preparation, Y.Y.; Writing-Review \& Editing, Y.Y., T.S.T., R.S., L.W., S.B., S.P., and E.J.S.

Funding: This research received no external funding.

Acknowledgments: none.

Conflicts of Interest: The authors declare no conflict of interest. 


\section{References}

1. Akresh IR. Overweight and obesity among foreign-born and U.S.-born Hispanics. Biodemography Soc Biol. 2008;54(2):183-199. doi:10.1080/19485565.2008.9989141.

2. Wolin KY, Colangelo LA, Chiu BCH, Gapstur SM. Obesity and immigration among Latina women. J Immigr Minor Heal. 2009;11(5):428-431. doi:10.1007/s10903-007-9115-1.

3. Barcenas $\mathrm{CH}$, Wilkinson A V, Strom SS, et al. Birthplace, years of residence in the United States, and obesity among Mexican-American adults. Obesity (Silver Spring). 2007;15(4):1043-1052. doi:10.1038/oby.2007.537

4. Oza-Frank R, Cunningham SA. The weight of US residence among immigrants: A systematic review. Obes Rev. 2010;11(4):271-280. doi:10.1111/j.1467-789X.2009.00610.x.

5. Albrecht SS, Diez Roux A V., Aiello AE, Schulz AJ, Abraido-Lanza AF. Secular trends in the association between nativity/length of US residence with body mass index and waist circumference among MexicanAmericans, 1988-2008. Int J Public Health. 2013;58(4):573-581. doi:10.1007/s00038-012-0414-5.

6. Guendelman SD, Ritterman-Weintraub ML, Fernald LCH, Kaufer-Horwitz M. Weight status of Mexican immigrant women: A comparison with women in Mexico and with US-born Mexican American women. Am J Public Health. 2013;103(9):1634-1640. doi:10.2105/AJPH.2012.301171.

7. Pérez-Escamilla R, Putnik P. The role of acculturation in nutrition, lifestyle, and incidence of type 2 diabetes among Latinos. J Nutr. 2007;137(4):860-870.

8. Nicolaou M, Van Dam RM, Stronks K. Acculturation and education level in relation to quality of the diet: A study of Surinamese South Asian and Afro-Caribbean residents of the Netherlands. J Hum Nutr Diet. 2006;19(5):383-393. doi:10.1111/j.1365-277X.2006.00720.x.

9. Pérez-Escamilla R. Acculturation, nutrition, and health disparities in Latinos. Am J Clin Nutr. 2011;93(5). doi:10.3945/ajcn.110.003467.

10. Neuhouser ML, Thompson B, Coronado GD, Solomon CC. Higher fat intake and lower fruit and vegetables intakes are associated with greater acculturation among Mexicans living in Washington State. J Am Diet Assoc. 2004;104(1):51-57. doi:10.1016/j.jada.2003.10.015.

11. Ayala GX, Baquero B, Klinger S. A systematic review of the relationship between acculturation and diet among Latinos in the United States: implications for future research. J Am Diet Assoc. 2008;108(8):13301344. doi:10.1016/j.jada.2008.05.009.

12. Monroe KR, Hankin JH, Pike MC, et al. Correlation of dietary intake and colorectal cancer incidence among Mexican-American migrants: the multiethnic cohort study. Nutr Cancer. 2003;45(2):133-147. doi:10.1207/S15327914NC4502_01.

13. Glanz K, Sallis JF, Saelens BE, Frank LD. Healthy nutrition environments: Concepts and measures. Am J Heal Promot. 2005;19(5):330-333. doi:10.4278/0890-1171-19.5.330.

14. Sharma S, Murphy SP, Wilkens LR, et al. Adherence to the food guide pyramid recommendations among African Americans and Latinos: Results from the multiethnic cohort. J Am Diet Assoc. 2004;104(12):18731877. doi:10.1016/j.jada.2004.08.033.

15. Beydoun MA, Gary TL, Caballero BH, Lawrence RS, Cheskin LJ, Wang Y. Ethnic differences in dairy and related nutrient consumption among US adults and their association with obesity, central obesity, and the metabolic syndrome. Am J Clin Nutr. 2008;87(6):1914-1925. doi:10.1016/j.drugalcdep.2008.02.002.A.

16. Drenowatz C, Shook RP, Hand G a, Hébert JR, Blair SN. The independent association between diet quality and body composition. Sci Rep. 2014;4:4928. doi:10.1038/srep04928.

17. Jennings A, Welch A, van Sluijs EMF, Griffin SJ, Cassidy A. Diet quality is independently associated with weight status in children aged 9-10 years. J Nutr. 2011;141(3):453-459. doi:10.3945/jn.110.131441.

18. Guenther PM, Casavale KO, Reedy J, et al. Update of the Healthy Eating Index: HEI-2010. J Acad Nutr Diet. 2013;113(4):569-580. doi:10.1016/j.jand.2012.12.016.

19. Tande DL, Magel R, Strand BN. Healthy Eating Index and abdominal obesity. Public Health Nutr. 2010;13(2):208-214. doi:10.1017/S1368980009990723.

20. Gao SK, Beresford SAA, Frank LL, Schreiner PJ, Burke GL, Fitzpatrick AL. Modifications to the healthy eating index and its ability to predict obesity: The multi-ethnic study of atherosclerosis. Am J Clin Nutr. 2008;88(1):64-69. 
431

432

433

434

435

436

437

438

439

440

441

442

443

444

445

446

447

448

449

450

451

452

453

454

455

456

457

458

459

460

461

462

463

464

465

466

467

468

469

470

471

472

473

474

475

476

477

478

479

480

481

482

483

21. Yoshida Y, Scribner R, Chen L, Broyles S, Phillippi S, Tseng TS. Diet quality and its relationship with central obesity among Mexican Americans: findings from National Health and Nutrition Examination Survey (NHANES) 1999-2012. Public Health Nutr 2017;20(7):1193-202. doi: 10.1017/S1368980016003190

22. Nicklas TA, Qu H, Hughes SO, He M, Wagner SE, Foushee HR, Shewchuk RM. Self-perceived lactose intolerance results in lower intakes of calcium and dairy foods and is associated with hypertension and diabetes in adults. Am J Clin Nutr. 2011; 94(1), 191-198. doi: 10.3945/ajcn.110.009860

23. Flegal KM, Carroll MD, Kit BK, Ogden CL. Prevalence of obesity and trends in the distribution of body mass index among US adults, 1999-2010. JAMA. 2012;307(5):491-497. doi:10.1001/jama.2012.39.

24. Bowie JV, Juon H-S, Cho J, Rodriguez EM. Factors associated with overweight and obesity among Mexican Americans and Central Americans: results from the 2001 California Health Interview Survey. Prev Chronic Dis. 2007;4(1):A10. doi:A10 [pii].

25. Wang Y, Beydoun MA. The obesity epidemic in the United States - Gender, age, socioeconomic, racial/ethnic, and geographic characteristics: A systematic review and meta-regression analysis. Epidemiol Rev. 2007;29(1):6-28. doi:10.1093/epirev/mxm007.

26. Wang J, Reininger BM, Cron S, Carroll D, Fisher-Hoch SP. Gender difference in obesity and preventive health behaviors in an US-Mexico border Hispanic cohort. International Journal of Exercise Science, 2002, $6(2)$.

27. National Center for Health Statistics. About National Health and Nutrition Examination Survey. http://www.cdc.gov/nchs/nhanes/about_nhanes.htm. Accessed April 20, 2018.

28. National Center for Health Statistics. National Health and Nutrition Examination, Anthropometry Procedure Manual. http://www.cdc.gov/nchs/data/nhanes/nhanes_07_08/manual_an.pdf. Accessed April 20, 2018.

29. Kandula NR, Diez-Roux A V., Chan C, et al. Association of acculturation levels and prevalence of diabetes in the multi-ethnic study of atherosclerosis (MESA). Diabetes Care. 2008;31(8):1621-1628. doi:10.2337/dc072182.

30. U.S. Department of Agriculture. The USDA Center for Nutrition Policy and Promotion. The Healthy Eating Index 2010. https://www.cnpp.usda.gov/healthyeatingindex. Accessed April 20, 2018.

31. U.S. Department of Health and Human Services. 2008 Physical activity guidelines for Americans. http://health.gov/paguidelines/guidelines/adults.aspx. Accessed May 20, 2016.

32. Ditlevsen S, Christensen U, Lynch J, Damsgaard MT, Keiding N. The mediation proportion: a structural equation approach for estimating the proportion of exposure effect on outcome explained by an intermediate variable. Epidemiology. 2005;16:114-120

33. Beydoun $\mathrm{M}$ a, Wang Y. How do socio-economic status, perceived economic barriers and nutritional benefits affect quality of dietary intake among US adults? Eur J Clin Nutr. 2008;62(3):303-313. doi:10.1038/sj.ejcn.1602700.

34. Winkleby MA, Albright CL, Howardpitney B, Lin J, Fortmann SP. Hispanic/White Differences in Dietary Fat Intake Among Low Educated Adults and Children. Prev Med (Baltim). 1994;23(4):465-473. doi:10.1006/pmed.1994.1064.

35. Liu J-H, Chu YH, Frongillo EA, Probst JC. Generation and acculturation status are associated with dietary intake and body weight in Mexican American adolescents. J Nutr. 2012;142(2):298-305. doi:10.3945/jn.111.145516.

36. Albertson AM, Reicks M, Joshi N, Gugger CK. Whole grain consumption trends and associations with body weight measures in the United States: results from the cross sectional National Health and Nutrition Examination Survey 2001-2012. Nutr J. 2016;15(1):8. doi:10.1186/s12937-016-0126-4.

37. Cioffi I, Santarpia L, Vaccaro A, et al. Whole-grain pasta reduces appetite and meal-induced thermogenesis acutely: a pilot study. Appl Physiol Nutr Metab = Physiol Appl Nutr Metab. 2016;41(3):277-283. doi:10.1139/apnm-2015-0446.

38. King DE. Dietary fiber, inflammation, and cardiovascular disease. Mol Nutr Food Res. 2005;49(6):594-600. doi:10.1002/mnfr.200400112.

39. Brown IJ, Tzoulaki I, Candeias V, Elliott P. Salt intakes around the world: Implications for public health. Int J Epidemiol. 2009;38(3):791-813. doi:10.1093/ije/dyp139.

40. Karppanen H, Mervaala E. Sodium Intake and Hypertension. Prog Cardiovasc Dis. 2006;49(2):59-75. doi:10.1016/j.pcad.2006.07.001. 
41. Pufal MA, Moulin CC, Casagrande DS, et al. Prevalence of overweight in children of obese patients: A dietary overview. Obes Surg. 2012;22(8):1220-1224. doi:10.1007/s11695-012-0641-0.

42. Ahuja JKC, Pehrsson PR, Haytowitz DB, et al. Sodium monitoring in commercially processed and restaurant foods. Am J Clin Nutr. 2015;101(3):622-631. doi:10.3945/ajcn.114.084954.

43. U.S. Centers for Disease Control and Prevention. Sodium and Food Sources. http://www.cdc.gov/salt/food.htm. Accessed May 20, 2016.

44. Lindberg NM, Stevens VJ. Immigration and weight gain: Mexican-American women's perspectives. J Immigr Minor Heal. 2011;13(1):155-160. doi:10.1007/s10903-009-9298-8.

45. Flores M, Macias N, Rivera M, et al. Dietary patterns in Mexican adults are associated with risk of being overweight or obese. J Nutr. 2010;140(10):1869-1873. doi:10.3945/jn.110.121533.

46. Jasti S, Lee CH, Doak C. Gender, acculturation, food patterns, and overweight in Korean immigrants. Am J Health Behav. 2011;35(6):734-745. doi:10.5993/AJHB.35.6.9.

47. Wolongevicz DM, Zhu L, Pencina MJ, et al. Diet quality and obesity in women: the Framingham Nutrition Studies. Br J Nutr. 2013;103(8):1223-1229. doi:10.1017/S0007114509992893.Diet.

48. Montez JK, Eschbach K. Country of Birth and Language Are Uniquely Associated with Intakes of Fat, Fiber, and Fruits and Vegetables among Mexican-American Women in the United States. J Am Diet Assoc. 2008;108(3):473-480. doi:10.1016/j.jada.2007.12.008.

49. Waijers PMCM, Feskens EJM, Ocké MC. A critical review of predefined diet quality scores. Br J Nutr. 2007;97(2):219-231. doi:10.1017/S0007114507250421.

50. Warren CS, Gleaves DH, Cepeda-Benito A, Fernandez MDC, Rodriguez-Ruiz S. Ethnicity as a protective factor against internalization of a thin ideal and body dissatisfaction. Int J Eat Disord. 2005;37(3):241-249. doi:10.1002/eat.20102.

51. Leung CW, Epel ES, Ritchie LD, Crawford PB, Laraia BA. Food Insecurity Is Inversely Associated with Diet Quality of Lower-Income Adults. J Acad Nutr Diet. 2014;114(12):1943-1953. doi:10.1016/j.jand.2014.06.353.

52. Martinez AD, Juon H, Levine DM, Lyford-Pike V, Peters S. The association between nutrition transition score and measures of obesity: Results from a cross-sectional study among Latina/o immigrants in Baltimore. Global Health. 2014;10:57. doi:10.1186/1744-8603-10-57.

53. Popkin BM, Adair LS, Ng SW, et al. Global nutrition transition and the pandemic of obesity in developing countries. Nutr Rev. 2012;70(1):3-21. doi:10.1111/j.1753-4887.2011.00456.x.

54. Fisher JO, Johnson RK, Lindquist C, Birch LL, Goran MI. Influence of body composition on the accuracy of reported energy intake in children. Obes Res. 2000;8:597-603. doi:10.1038/oby.2000.77.

55. Larson NI, Neumark-Sztainer D, Hannan PJ, Story M. Family Meals during Adolescence Are Associated with Higher Diet Quality and Healthful Meal Patterns during Young Adulthood. J Am Diet Assoc. 2007;107(9):1502-1510. doi:10.1016/j.jada.2007.06.012.

56. Institute of Medicine, Dietary Reference Intakes for Water, Potassium, Sodium, Chloride, and Sulfate, 2005. https://www.nap.edu/read/10925/chapter/1. Accessed April 20, 2018 